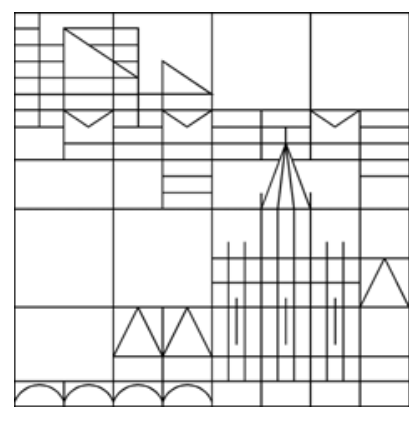

POD-Based Multiobjective Optimal Control of Time-Variant Heat Phenomena

\author{
Stefan Banholzer \\ Eugen Makarov \\ Stefan Volkwein
}

Konstanzer Schriften in Mathematik

Nr. 370, November 2017

ISSN 1430-3558

Konstanzer Online-Publikations-System (KOPS)

URL: http://nbn-resolving.de/urn:nbn:de:bsz:352-2--1er2jsajujq0x2

(C) Fachbereich Mathematik und Statistik

Universität Konstanz

Fach D 197, 78457 Konstanz, Germany 



\title{
POD-Based Multiobjective Optimal Control of Time-Variant Heat Phenomena
}

\author{
Stefan Banholzer, Eugen Makarov and Stefan Volkwein \\ University of Konstanz, Department of Mathematics and Statistics, \\ Universitätsstraße 10, D-78457 Konstanz, Germany \\ Stefan.Banholzer@uni-konstanz.de*, Stefan.Volkwein@uni-konstanz.de
}

\begin{abstract}
In the present paper, a multiobjective optimal control problem governed by a heat equation with time-dependent convection term and bilateral control constraints is considered. For computing Pareto optimal points and approximating the Pareto front, the reference point method is applied. As this method transforms the multiobjective optimal control problem into a series of scalar optimization problems, the method of proper orthogonal decomposition (POD) is introduced as an approach for model-order reduction. New strategies for efficiently updating the POD basis in the optimization process are proposed and tested numerically.
\end{abstract}

\section{Introduction}

Many optimization problems in applications can be formulated using several objective functions, which are conflicting with each other. This leads to the notion of multiobjective or multicriterial optimization problems; cf. [4,9,12]. One prominent example is given by an energy efficient heating, ventilation and air-conditioning (HVAC) operation of a building with conflicting objectives such as minimal energy consumption and maximal comfort; cf. [6,8].

In this paper we apply the reference point method [11] in order to transform a bicriterial optimal control problem into a sequence of scalar-valued optimal control problems and solve them using well-known optimal control techniques; see [13]. We build on and extend previous results obtained in [2], where a linear convection-diffusion equation was considered. In addition, we allow the convection term to be time-dependent here.

By using the a-posteriori error estimate [2, Theorem 9] we develop a new strategy for updating the POD basis while computing the Pareto front such that the error stays always below a certain predefined threshold. In our numerical examples we compare the strategy with the simple basis extension algorithm in [2, Algorithm 3]. Moreover, we propose a method to choose an efficient initial number of POD basis functions.

The paper is organized in the following manner: In Section 2 we present the state equation and the bicriterial optimal control problem. The reference point method and how to apply it to the problem at hand is explained in

\footnotetext{
* S. Banholzer gratefully acknowledges support by the German DFG-Priority Pro-
} gram 1962 and by the Landesgraduiertenförderung of Baden-Württemberg. 
Section 3. Moreover, the POD method is briefly introduced to gain a speedup in the solution process. Section 4 contains the numerical experiments and in Section 5 we draw a conclusion.

\section{Problem formulation}

The state equation: Let $\Omega \subset \mathbb{R}^{d}$ with $d \in\{2,3\}$ be a bounded domain with Lipschitz-continuous boundary $\Gamma$. We choose $m$ non-empty, pairwise disjoint subsets $\Omega_{1}, \ldots, \Omega_{m}$ of the domain $\Omega$. For a given end time $T>0$, we set $Q:=(0, T) \times \Omega$ and $\Sigma:=(0, T) \times \Gamma$. The state equation is then given by the following diffusion-convection equation with homogeneous Neumann boundary conditions:

$$
\begin{aligned}
y_{t}(t, x)-\kappa \Delta y(t, x)+\beta(t, x) \cdot \nabla y(t, x) & =\sum_{i=1}^{m} u_{i}(t) \chi_{i}(x) & & \text { in } Q, \\
\frac{\partial y}{\partial \boldsymbol{n}}(t, x) & =0 & & \text { on } \Sigma, \\
y(0, x) & =y_{0}(x) & & \text { in } \Omega .
\end{aligned}
$$

In (1a) the constant $\kappa>0$ is the diffusion coefficient and the time-dependent advection $\beta$ is supposed to be in $L^{\infty}\left(Q ; \mathbb{R}^{d}\right)$. Furthermore, the function $\chi_{i}$ is given by the characteristic function of the set $\Omega_{i}$ for all $i=1, \ldots, m$. For the control variable $u=\left(u_{1}, \ldots, u_{m}\right)$ we assume $u \in U=L^{2}\left(0, T ; \mathbb{R}^{m}\right)$. Finally in (1c), $y_{0} \in H=L^{2}(\Omega)$ is a given initial temperature. To set the framework for the weak formulation of (1), we define the Hilbert space $V=H^{1}(\Omega)$ equipped with the standard inner product. The space

$$
Y=W(0, T)=\left\{\phi \in L^{2}(0, T ; V) \mid \phi_{t} \in L^{2}\left(0, T ; V^{\prime}\right)\right\}
$$

endowed with the canonical inner product is a Hilbert space; see, e.g. [3]. With similar arguments as in [1, Section 5.1] it is possible to show that for each tuple $\left(u, y_{0}\right) \in U \times H$ there is a unique weak solution $y \in Y$ of (1). Furthermore, the solution can be written as $y=\hat{y}+\mathcal{S} u$, where $\hat{y} \in Y$ is the weak solution of (1) for the tuple $\left(0, y_{0}\right)$ and the linear operator $\mathcal{S}: U \rightarrow Y$ is given such that $\mathcal{S} u$ is the weak solution of (1) to the tuple $(u, 0)$.

The bicriterial optimal control problem: For a given desired temperature $y_{Q} \in L^{2}(0, T ; H)$ we introduce the cost functional

$$
J: Y \times U \rightarrow \mathbb{R}^{2}, \quad J(y, u)=\left(\frac{1}{2}\left\|y-y_{Q}\right\|_{L^{2}(0, T ; H)}^{2}, \frac{1}{2}\|u\|_{L^{2}\left(0, T ; \mathbb{R}^{m}\right)}^{2}\right)^{\top} .
$$

Defining the set $U_{\mathrm{ad}}=\left\{u \in U \mid u_{a} \leq u \leq u_{b}\right.$ in $\left.[0, T]\right\}$ for given $u_{a}, u_{b} \in U$ with $u_{a} \leq u_{b}$ in $[0, T]$, the bicriterial optimal control problem reads

$$
\min J(y, u) \quad \text { s.t. } \quad(y, u) \in\left\{(\tilde{y}, \tilde{u}) \in Y \times U_{\text {ad }} \mid \tilde{y}=\hat{y}+\mathcal{S} \tilde{u}\right\} .
$$


Since $\mathcal{S}$ is well-defined, we define the reduced cost function $\hat{J}: U \rightarrow \mathbb{R}^{2}$, $\hat{J}(u)=J(\hat{y}+\mathcal{S} u, u)$ and investigate the reduced formulation of (2) in this paper:

$$
\min \hat{J}(u) \quad \text { s.t. } \quad u \in U_{\text {ad }} .
$$

Problem (3) involves the minimization of a vector-valued function with two objectives. This is done by using the concept of Pareto optimality; cf. [4].

Definition 1. The point $\bar{u} \in U_{\text {ad }}$ is called Pareto optimal for (3) if there is no other control $u \in U_{\text {ad }} \backslash\{\bar{u}\}$ with $\hat{J}_{i}(u) \leq \hat{J}_{i}(\bar{u}), i=1,2$, and $\hat{J}(u) \neq \hat{J}(\bar{u})$.

\section{The reference point method}

The theoretical and numerical aim in solving a bicriterial optimization problem is to get an approximation of the Pareto set and the Pareto front, respectively, which are given by

$$
P_{s}=\left\{u \in U_{\text {ad }} \mid u \text { is Pareto optimal }\right\} \subset U \text { and } \quad P_{f}=\hat{J}\left(P_{s}\right) \subset \mathbb{R}^{2} .
$$

The scalarization method, in which the bicriterial function is transformed into a scalar function and then minimized using well-known techniques from scalar optimization, is one of the most popular approaches to tackle this problem, see e.g. $[5,9,12]$. The idea is that by choosing different scalarizations, both the Pareto set and the Pareto front can be approximated. One particular scalarization method is the (Euclidean) reference point method, which was previously used in $[10,11]$. Given a reference point $z \in P_{f}+\mathbb{R}_{\leq}^{2}=\{z+x \mid$ $z \in \mathcal{P}_{f}$ and $\left.x \in \mathbb{R}_{\leq}^{2}\right\}$ the distance function

$$
F_{z}: U \rightarrow \mathbb{R}, \quad F_{z}(u)=\frac{1}{2}\left(\hat{J}_{1}(u)-z_{1}\right)^{2}+\frac{1}{2}\left(\hat{J}_{2}(u)-z_{2}\right)^{2}
$$

measures the Euclidean distance between $\hat{J}(u)$ and $z$ for a given $u \in U$. The idea is that by solving the minimization problem

$$
\min F_{z}(u) \text { s.t. } u \in U_{\mathrm{ad}},
$$

we get a Pareto optimal point for (3). The following theorem, which is taken from [1, Theorem 3.35], guarantees this property for the problem at hand.

Theorem 2. Let $z \in P_{f}+\mathbb{R}_{<}^{2}$ be a reference point. Then (4) has a unique solution $\bar{u} \in U_{\mathrm{ad}}$, which is Pareto optimal for (3).

The algorithmic approach to approximate $P_{f}$, which we consider in this paper, first computes the two boundary points of the Pareto front. These are given by the minimizers of $\hat{J}_{1}$ and $\hat{J}_{2}$, respectively. In our case we have to regularize the minimization of $\hat{J}_{1}$ because $\hat{J}_{1}$ is only strictly, but not strongly convex. Therefore, we minimize $\hat{J}_{1}+\alpha \hat{J}_{2}$ with a small weight $0<\alpha \ll 1$. We 
always choose the minimizer of $\hat{J}_{1}$ as a starting point. Given a Pareto optimal point the algorithm generates a new reference point following $P_{f}$ from top to bottom, and then solves the respective reference point problem. This procedure is repeated until the end of $P_{f}$ is reached. The exact scheme for computing the reference points along with a more detailed description of the algorithm can be found in [1, Section 3.4] and [2, Section 6].

When implementing this algorithm, (4) has to be solved repeatedly for different reference points $z \in P_{f}+\mathbb{R}_{<}^{2}$. Each solve of (4) requires multiple solves of (1) and an adjoint equation (cf. [13, Section 3.6]) which is often computationally too costly when using a standard Finite Element (FE) method. Thus, it is reasonable to apply model-order reduction to reduce the computational effort. We use the well-known POD method; cf. [7]. In [1,2] the procedure for our problem at hand is explained. Here, we just want to introduce some notations: Given a POD basis of rank $\ell$, we define the set $V^{\ell}=\operatorname{span}\left\{\psi_{1}, \ldots, \psi_{\ell}\right\}$ and the solution operator $\mathcal{S}^{\ell}: U \rightarrow H^{1}\left(0, T ; V^{\ell}\right)$ of the POD solution of the $u$-dependent part of the state equation. The POD approximation of $\hat{J}$ is defined as $\hat{J}^{\ell}(u)=J\left(\hat{y}+\mathcal{S}^{\ell} u, u\right)$. Then, (4) is replaced by

$$
\min F_{z}^{\ell}(u)=\frac{1}{2}\left(\hat{J}_{1}^{\ell}(u)-z_{1}\right)^{2}+\frac{1}{2}\left(\hat{J}_{2}^{\ell}(u)-z_{2}\right)^{2} \quad \text { s.t. } \quad u \in U_{\text {ad }}
$$

with $\hat{J}_{2}^{\ell}(u)=\hat{J}_{2}(u)$. For a given reference point $z \in \mathcal{P}_{f}+\mathbb{R}_{\leq}^{2}$ we denote the optimal solutions to (4) and (5) by $\bar{u}_{z}$ and $\bar{u}_{z}^{\ell}$, respectively.

\section{Numerical Results}

In our numerical tests we consider the bicriterial optimal control problem presented in Section 2. We have $\Omega=(0,1)^{2} \subset \mathbb{R}^{2}$ and choose $T=1$. The diffusion parameter is given by $\kappa=0.5$ and for the convection term in (1a) we use $\beta(t, x)=c_{b} \tilde{\beta}(t, x)$ for all $(t, x) \in Q$, where $\tilde{\beta}$ is a non-stationary solution of a Navier-Stokes equation and $c_{b} \geq 0$ is a parameter to control the strength of the convection; cf. Fig. 1. We impose a floor heating of the whole room with $m=4$ uniformly distributed heaters in the domains $\Omega_{1}=$ $(0,0.5)^{2}, \Omega_{2}=(0,0.5) \times(0.5,1), \Omega_{3}=(0.5,1) \times(0,0.5)$ and $\Omega_{4}=(0.5,1)^{2}$. The bilateral control constraints are $u_{a}=0$ and $u_{b}=3$. Finally, we choose $y_{0}=16$ and $y_{Q}(t, x)=16+2 t$ for all $(t, x) \in Q$. All computations were carried out on a MacBook Pro 13 (middle 2012) with $2.5 \mathrm{GHz}$ Intel Core i5 and 4GB RAM.

Test 1: We solve (3) for $c_{b}=1$. Then, $P_{f}$ is smoothly approximated by 52 Pareto optimal points; cf. Fig. 2. Hereby, $P_{f}$ ranges from $P^{1}=(0.0199,4.1)$, which is computed with the weighted-sum method with weight $\alpha=0.02$, to $P^{52}=(0.6667,0)$. Thus, the desired temperature can be achieved quite closely in the upper part of $P_{f}$. The four optimal controls for $P^{1}$ can be seen in right plot of Fig. 2. As in the case of a time-independent convection term all four controls adapt to the air flow, which goes from the top left 


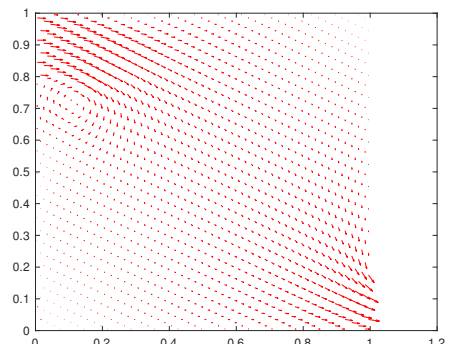

(a) $t=0.01$

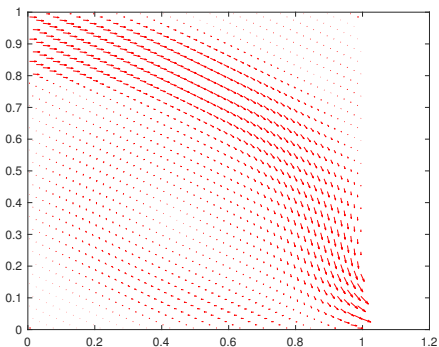

(b) $t=0.5$

Fig. 1. Time-dependent convection $\beta(t, x)$ at two time instances.
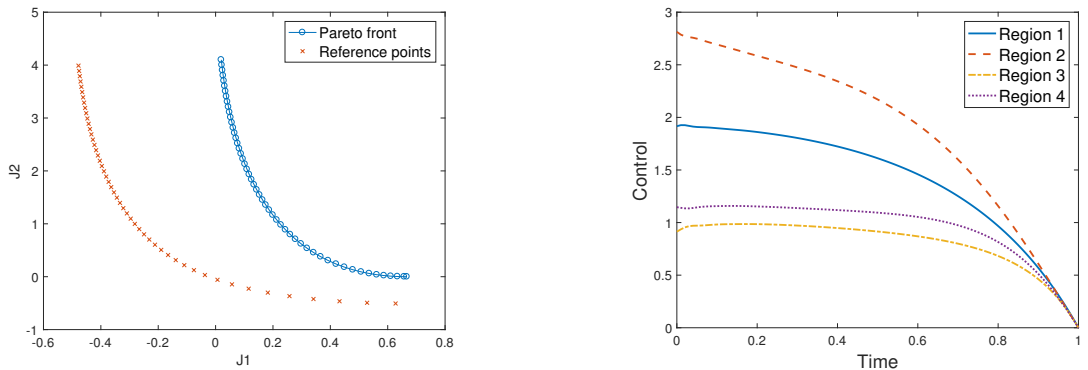

Fig. 2. Test 1: Pareto front (left); optimal control $\bar{u}^{1}$ corresponding to $P^{1}$ (right).

corner of the room to the right bottom corner by using different heating strategies. Furthermore, one can observe a slightly wavy behaviour of the optimal controls at the beginning. This is due to the temporal changes in the dynamics of the system caused by a vortex moving over time from the top left corner to the middle of the room. Another interesting aspect is that in the comparison to the time-independent case (by taking average over the time) the system with time-dependent convection only needs about $10 \%$ more computation time although the latter case adds dynamics to the optimal control problem, which are more difficult to handle numerically.

Test 2: Now we use an adaptive POD basis extension algorithm, which was introduced in [2, Algorithm 3], in order to investigate the influence of the time-dependent convection term on the number of needed POD basis functions. As a measure for the error between $u_{z}^{\ell}$ and $\bar{u}_{z}^{\ell}$ induced by the POD approximation we use the a-posteriori estimate [2, Theorem 9] and set the upper bound of the acceptable error to $\mu=4 \cdot 10^{-4}$, as well as the initial number of POD basis functions to $\ell_{\text {init }}=5$. Choosing the minimizer of $\hat{J}_{1}$ as starting point we observe that 24 POD basis functions are needed to compute 
the whole Pareto front in the desired approximation quality. Thereby, all 19 basis extensions are conducted on $P^{2}$. For comparison, in [2] was shown that for a very similar problem with a time-independent convection term only 15 POD basis functions are sufficient to achieve the same quality. This is due to the fact that a time-dependency of a convection adds more complex dynamics to the system which are hard to capture by using only one fixed POD basis. To tackle this problem we propose a POD basis improving strategy. Algorithm 1 shows the routine for computing the $n$-th Pareto optimal point. In this paper we investigate two strategies for determining $\ell$ after each basis update. In

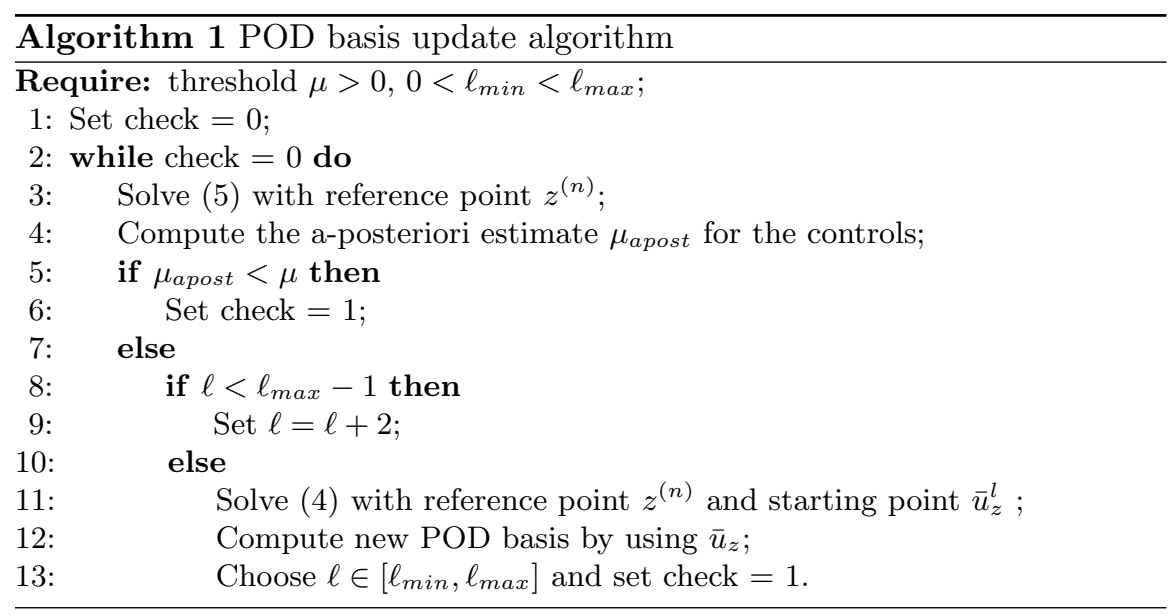

the first case, we set $\ell=\ell_{\min }$. In the second case, we choose $\ell$ by observing the convergence rates in the control space; cf. [7, Theorem 1.49]. Namely, by choosing $\ell \in\left[\ell_{\min }, \ell_{\max }\right]$ such that

$$
\sum_{i=\ell+1}^{\ell_{\max }} \lambda_{i}\left\|\psi_{i}-\mathcal{P}_{H}^{\ell} \psi_{i}\right\|_{H^{1}(\Omega)}^{2}<\varepsilon
$$

for an $\varepsilon<\mu$ holds, where $\mathcal{P}_{H}^{\ell}$ denotes the orthogonal projection onto $V^{\ell}$, $\left\{\psi_{i}\right\}_{i \in \mathbb{N}}$ is a POD basis and $\left\{\lambda_{i}\right\}_{i \in \mathbb{N}}$ are the corresponding eigenvalues. The results for $\varepsilon=5 \cdot 10^{-3} \mu$ and $\ell_{\max }=22$ are presented in Fig. 3 and Table 1. The value of $\ell_{\min }$ is set to 10 and to 6 by the first and second strategy, respectively. Using the second strategy Algorithm 1 yields the best results with regard to the CPU time. Thus, (6) estimates quite well how many POD basis functions would have been necessary in order to compute the current Pareto point. As a result only 2 basis extensions on the points $P^{2}$ and $P^{3}$ are needed. Using the first strategy, 11 basis extensions are necessary as 10 POD basis function are not enough even after a basis update. Hence, a lot of avoidable basis extensions are done. In both cases one basis update is 


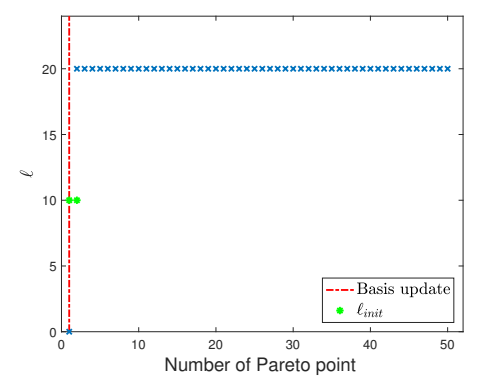

(a) $\ell=10$ fixed

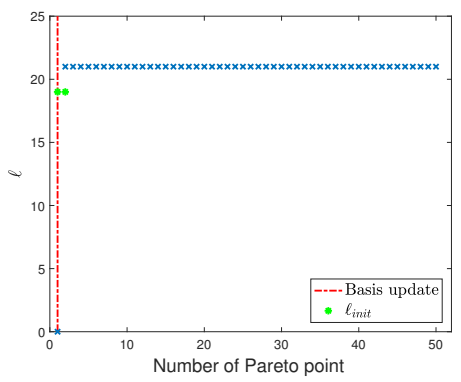

(b) $6 \leq \ell \leq 22$ adaptive

Fig. 3. Number of used POD basis functions in Algorithm 1 using $\varepsilon=0.5 \cdot 10^{-2} \mu$.

Table 1. Test 2: Results for $\varepsilon=0.5 \cdot 10^{-2} \mu$

\begin{tabular}{lccc}
\hline & CPU time & \#Basis extensions & \#Basis updates \\
\hline Full system & 240.25 & - & - \\
Basis extension with $\ell_{\text {init }}=5$ & 51.07 & 19 & - \\
Alg. 1 with fixed $\ell=10$ & 49.53 & 11 & 1 \\
Alg. 1 with adaptive $\ell \in[6,22]$ & 44.82 & 2 & 1 \\
Alg. 1 with adaptive $\ell \in[6,20]$ & 59.51 & 0 & 6 \\
\hline
\end{tabular}

conducted. However, the performance of the Algorithm 1 depends strongly on the choice of $\ell_{\max }$. Decreasing $\ell_{\max }$ to 20 increases the CPU time by $33 \%$ as 6 basis updates are needed in this case. Furthermore, $\varepsilon$ has to be chosen appropriately to avoid unnecessary basis extensions in the second case.

Test 3: Now we increase the strength of the convection term $c_{b}$ to 2 and run the basis extension algorithm with the same settings. Surprisingly, in the basis extension algorithm [2, Algorithm 3] only 25 POD basis functions are needed to compute the whole Pareto front, although there are significant changes in the behaviour of the controls. However, as expected, increasing the strength of the convection term increases heavily the number of basis updates in Algorithm 1 for the same values of $\ell_{\max }$ and thus the computation time.

\section{Conclusion}

In the present paper we show how including a time-dependent advection term into the state equation influences the results of the bicriterial optimal control problem (2). As expected the time-dependence adds dynamics to the system which cannot be captured that easily by a single POD basis. Therefore, by introducing a new POD update strategy we are able to save about $15 \%$ of the CPU time in comparison to the basis extension algorithm in $[2$, Algorithm $3]$. 


\section{References}

1. S. BAnHolzer, POD-Based Bicriterial Optimal Control of ConvectionDiffusion Equations, Master thesis, University of Konstanz, Department of Mathematics and Statistics, 2017, see http://nbn-resolving.de/ urn:nbn:de:bsz:352-0-421545.

2. S. Banholzer, D. Beermann and S. Volkwein, POD-based error control for reduced-order bicriterial PDE-constrained optimization, Annu. Rev. Control 44 (2017), 226-237.

3. R. Dautray And J.-L. Lions, Mathematical Analysis and Numerical Methods for Science and Technology. Volume 5: Evolution Problems I, Springer-Verlag, Berlin, 2000.

4. M. Ehrgotт, Multicriteria Optimization, Springer Verlag, Berlin, 2005.

5. G. EichFELder, Adaptive Scalarization Methods in Multiobjective Optimization, Springer Verlag, Berlin Heidelberg, 2008

6. K. F. Fong, V. I. HAnBy, And T.-T. CHOW, HVAC system optimization for energy management by evolutionary programming, Energy and Buildings, $38: 220-231,2006$.

7. M. Gubisch And S. Volkwein, Proper orthogonal decomposition for linearquadratic optimal control, In P. Benner, A. Cohen, M. Ohlberger, and K. Willcox (eds.), Model Reduction and Approximation: Theory and Algorithms. Pages 5-66, SIAM, Philadelphia, PA, 2017.

8. A. Kusiak, F. TAng, AND G. Xu., Multi-objective optimization of HVAC system with an evolutionary computation algorithm, Energy, 36:2440-2449, 2011.

9. K. Miettinen, Nonlinear Multiobjective Optimization International Series in Operations Research \& Management Science, Springer, 1998.

10. S. Peitz, S. Oder-BlÖBAum And M. Dellnitz, Multiobjective optimal control methods for fluid flow using reduced order modeling. 24th Congress of Theoretical and Applied Mechanics (ICTAM), 21-26 August 2016, Montreal, Canada, see http://arxiv.org/pdf/1510.05819v2.pdf.

11. C. Romaus, J. Böcker, K. Witting, A. Seifried, and O. ZnamenSHCHYKOV, Optimal energy management for a hybrid energy storage system combining batteries and double layer capacitors, IEEE, pages 1640-1647, San Jose, CA, USA, 2009.

12. W. StadLER, Multicriteria Optimization in Engineering and in the Sciences, Plenum Press, New York, 1988.

13. F. Tröltzsch, Optimal Control of Partial Differential Equations: Theory, Methods and Applications, AMS American Mathematical Society, 2nd ed., 2010. 